\title{
TEXTURE DEVELOPMENT AND ANISOTROPIC PHOTOELASTIC PROPERTIES IN ROLLED SILVER CHLORIDE
}

\author{
P. DIETZ and H. GIELEßEN \\ Institut für Maschinenwesen (Institute of Mechanical Engineering), Technische \\ Universität Clausthal, Robert-Koch-Straße 32, D-38678 Clausthal-Zellerfeld, \\ Germany, email: gielesen@imw.tu-clausthal.de
}

(Received 5 October 1994; in final form 10 November 1994)

\begin{abstract}
Experimental and theoretical studies have been carried out in order to relate elastic anisotropy to optical anisotropy by means of photoelasticity. The fundamentals of anisotropic photoelasticity have been described. Specimens of monocrystalline and polycrystalline silver chloride have been submitted to tensile stress and relative retardation and extinction angles observed in polarized monochromatic light to show conformity to the theories which quantitatively relate the state of stress and optical phenomena. Textures of cold-rolled as well as of recrystallized silver chloride specimens were determined with an X-ray goniometer. Texture determining parameters such as degree of rolling and recrystallisation time and temperature have been varied. Textures found in silver chloride after various processing have been characterized.
\end{abstract}

KEY WORDS: Silver chloride, photoplasticity, photoelasticity, optical anisotropy, transparent metal, texture modelling.

\section{INTRODUCTION}

Why are mechanical engineers interested in texture and anisotropy? And why are they interested in a material like silver chloride which is of absolutely no significance as a material for machine parts? In the field of mechanical engineering there is one main task beside designing and that is the determination of mechanical stresses in mechanical parts. There are different tools in the toolbox of mechanical engineering which can be used to achieve this. First there is the analytical approach which is suitable when the geometry and the load conditions are simple. Numerical methods like FEM or BEM are most often used now that highly developed software and computation capabilities are available at a comparably low cost. However, a high standard of experience is necessary to achieve results which can be looked upon as being correct. These methods are sensitive to the chosen type of elements and to the elementation of the mesh.

In the range of experimental methods the only widely used method when wholefield measurements are to be made is photoelasticity. This method utilizes the effect of stress birefringence that occurs in every transparent material whether it is crystalline or not. Analogies have been developed which permit use of transparent models, which, when loaded by external or internal forces, also allow investigation of stresses in inner 
parts of the structure. The major drawback of the method is the need to prepare a model of the real part if determination of not only the surface stresses is needed.

All of the commonly used model materials have a non-crystalline structure so that they are only suitable for studying problems of the elasticity theory of a homogeneous and isotropic body as they are homogeneous and isotropic in their structure, mechanical and optical properties themselves. The majority of materials used in engineering, however, are metals and thus of crystalline structure. Anisotropic properties have normally been induced by various types of thermal and mechanical processing these parts have usually been submitted to. Another drawback of photoelastic materials, which are mostly polymer resins of different kinds, is that their mechanical behaviour above the yielding point is totally different from the yield of metals. Plastic deformation of polymers takes place as breaking, forming and rearranging of the polymeric chains. There are no slipplanes, twinning or work-hardening. Furthermore, deformation is depending on load times and strain rates. For these reasons there was the need of a transparent material exhibiting textural effects, slip-planes, work-hardening, recrystallisation, workability and sufficient stress-optical sensitivity. Materials to meet all these requirements had to be crystalline. By Tamman (1932) silver and thallium halide salts were described for the first time to be suitable for photoelastic studies. Stepanov (1956a), who was the first one to give a description of the preparation of silver chloride specimens for photoelastic purposes formed the term "transparent metals" for these substances. As they are of cubic structure they are not naturally doubly refracting and their birefringence gives a direct representation of magnitude and direction of internal stresses. These "transparent metals" can be rolled, forged, cut, annealed and recrystallized just like metals. Anisotropic optical behaviour caused by textures turns up as a direct consequence of mechanical treatment just like mechanical anisotropy occurs in metals after mechanical treatment. However the early researchers were interested in textural effects only because they wanted to avoid them. A method was suggested how to obtain nearly texturefree polycrystalline specimens by Stepanov (1956b) which was developed further by Weber (1986).

We started our research on silver chloride in the late 70 s when there was the need for a stress-optical material with high plasticity to simulate metal forming processes. At present time our work concentrates on the creation of optical anisotropy in silver chloride that can be related to mechanical anisotropy in rolled metal sheets and to formulate the fundamentals of photoelasticity with textured material.

\section{NEW DEMANDS ON PHOTOELASTICITY}

The approach of an anisotropic photoelasticity requires the combination of conventional photoelasticity with the laws of crystal optics in consideration of the texture in the model as well as in the real part. Conventional photoelasticity deals with amorphous, molecular or isotropic materials whereas the laws of crystal optics describe the optical behaviour of single crystals. The polycrystalline aggregate can be described by the orientation distribution function (ODF) from which different physical properties can be calculated.

The real machine part that is suitable for these investigations of mechanical anisotropy is preferably made of steel because aluminium has only very small elastic anisotropy. The mechanical properties are influenced by the texture of the material which is again caused by the state of deformation. The texture is described by the coefficients of the 


$$
\text { single crystal } \Rightarrow \text { crystal optics }
$$

isotropic polycrystal $\Rightarrow$ conventional photoelasticity

textured polycrystal $\Rightarrow$ anisotropic experimental stress analysis

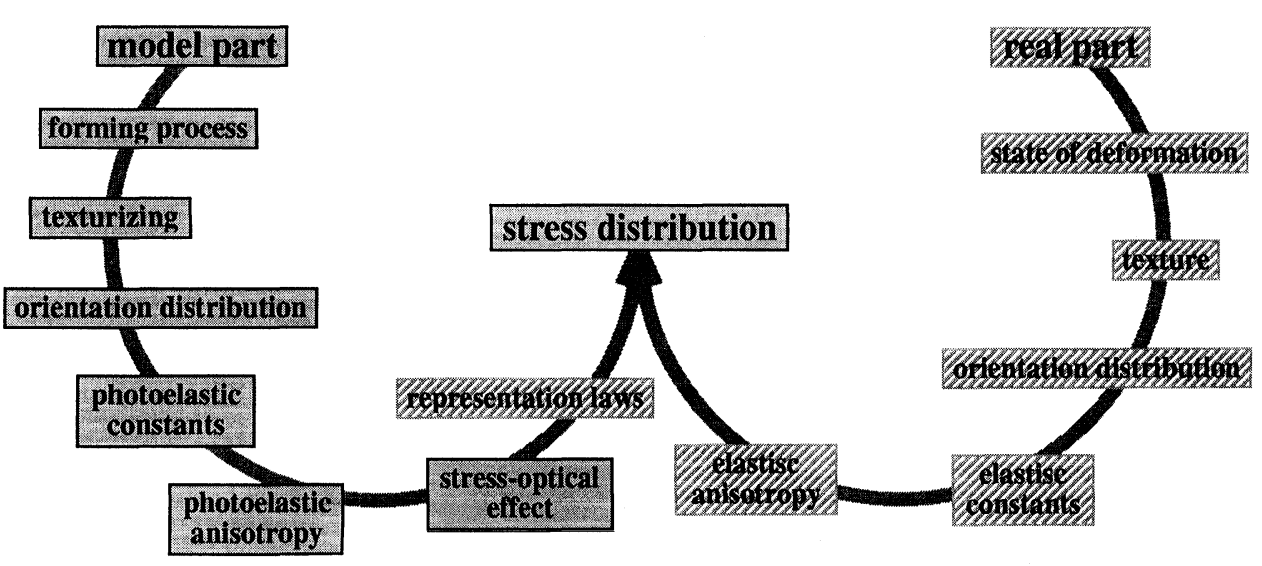

Figure 1 Overview of anisotropic photoelasticity.

orientation distribution function. With the elastic constants, that are known very well for most materials, the macroscopic elastic anisotropy can be calculated from the constants of the ODF. Together with an external load or known residual stresses this yields the stress or strain distribution.

To simulate the stress distribution of the machine part by means of photoelasticity a model of the part must be made of silver chloride. By forming processes like extruding and rolling and recrystallisation processes textures of different kind and different degrees can be obtained. The final shape of the model is best achieved by machining after forming. The texture coefficients have to be determined and together with the photoelastic or piezooptic constants, of which there are three in the case of silver chloride, the photoelastic behaviour of the textured material can be calculated. Stressoptical behaviour in this sense means changes of the refraction indices of two polarized lightwaves whose directions of polarization are perpendicular. Superposition of the two waves yields interferences which can be measured and from which the state of stress in the model can be calculated. By application of appropriate representation laws the state of stress of the real part can be determined. The following treatise will deal with the topics in the grey shaded boxes in Figure 1.

\section{GLIDE MECHANISM IN SILVER CHLORIDE}

To be able to discuss the anisotropic properties of silver chloride, the mechanism of deformation under load has to be clarified. The raw material for the specimens that 
has been produced in a zone melting furnace is first extruded and then rolled to obtain a fine grained structure. Caused by the deformation fine glide lines appear on the surface of the specimen as is shown in Figure 2. These glide lines are similar to those which can be seen on polished metallic surfaces after deformation. By Nye (1949) it was shown that these lines represent fine steps on the surface and thus are caused by glide and not by twinning. The occurrence of glide lines depends on the orientation of the grains relative to the surface. Figure 2 shows two neighbouring grains in one of which the normal of the glide plane forms a small angle with the surface. In the other grain the glide plane is nearly parallel to the surface so that glide lines do not show up. Another typical phenomenon is the appearance of birefringent bands when the specimen is observed in polarized light (Figure 3). These bands are a consequence of remaining stresses between neighbouring glide zones after deformation. Their appearance again depends on the inclination of the glide planes to the surface of the specimen. The distance of the birefringent bands is small at large angles but at small angles the bands might disappear due to mutual extinction because they are overlapping. In this case two systems of glide lines can be seen with a system of birefringent bands orientated parallel to the bisector of the angle between the glide systems.

Nye (1949) found by optical determination of the orientation of the glide lamellae and orientation measurement of the grains by the Laue method that the glide planes are in most cases near [111] (Figure 4). Glide direction was found to be $\langle 110\rangle$ which is in accordance with fcc metals.

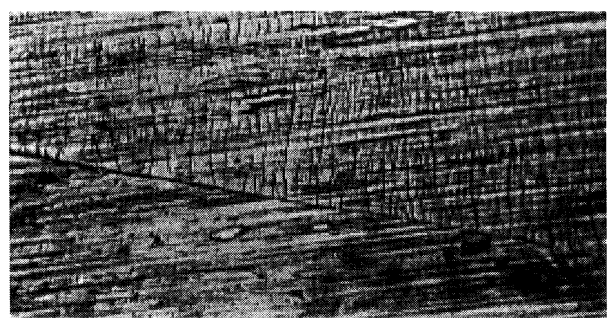

Figure 2 Glide lines in $\mathrm{AgCl}$.

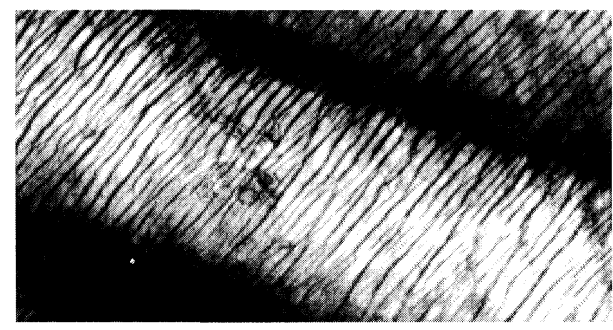

Figure 3 Birefringent bands in $\mathrm{AgCl}$.

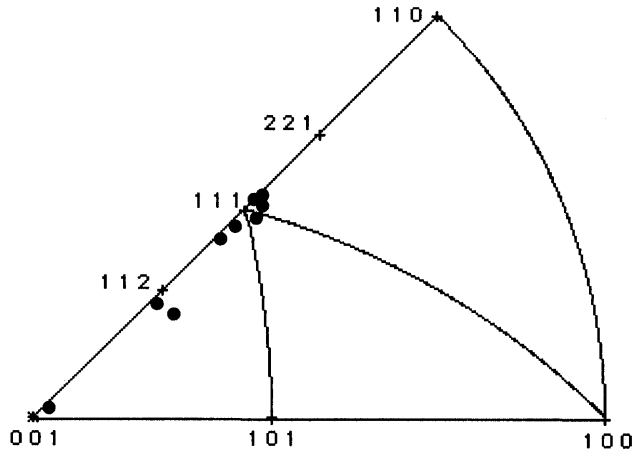

Figure 4 Poles of glide planes of several grains. 


\section{TEXTURE DEVELOPMENT IN ROLLED SILVER CHLORIDE}

From the structure of the material it can be concluded that textures as a consequence of rolling or extruding should occur. Different authors reported optical anisotropy of silver chloride and the existence of texture was suggested. However, this was never investigated further until now. Pole figures of our first experiments showed only weak textural effects so systematical investigations were made.

The raw material produced in a Brigdman-Stockbarger zone-melting furnace had a diameter of $32 \mathrm{~mm}$ and a length of approximately $80 \mathrm{~mm}$. All specimens which were used for rolling were obtained by extruding with a cross section of $30 \times 12 \mathrm{~mm}$. They showed quite uniformly sized grains with an average diameter of $100 \mu \mathrm{m}$. Rolling took place in steps of $20 \%, 40 \%, 60 \%, 80 \%, 90 \%, 95 \%$ and $98 \%$ reduction. Three different temperatures, $23^{\circ} \mathrm{C}, 0^{\circ} \mathrm{C}$, and $-35^{\circ} \mathrm{C}$ were used for rolling. Figure 5 shows grain sizes at various degrees of rolling at $-35^{\circ} \mathrm{C}$. Significant elongation of the grains began at about $40 \%$ and reached its maximum at $60 \%$. Rolling at higher temperatures shifted these values to $30 \%$ and $40 \%$ respectively. There was only little difference between the experiments at $23^{\circ} \mathrm{C}$ and $0^{\circ} \mathrm{C}$. There is a maximum of the grain size at $80 \%$ rolling (elevated temperatures: $60 \%-80 \%$ ) of about $0.2 \mathrm{~mm}$.

The original intention was to obtain a smaller grain by rolling because this is more suitable for optical investigations. The effect of increasing grain sizes is contrary to literature and it is believed that this is caused by a probably higher purity of our material. Evidently dynamic recrystallisation took place during rolling which in turn

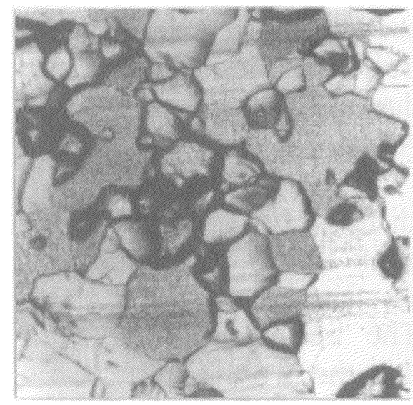

$\varphi=0 \%$

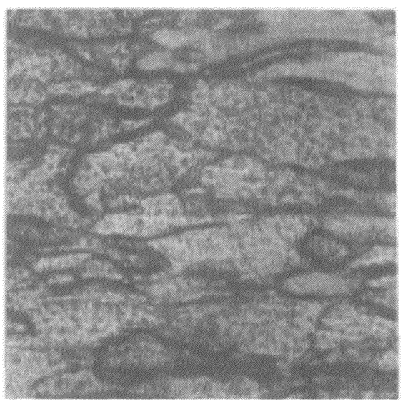

$\varphi=60 \%$

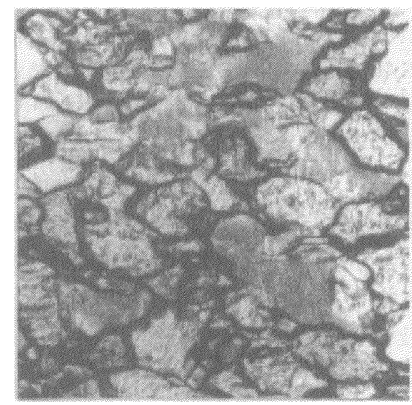

$\varphi=20 \%$

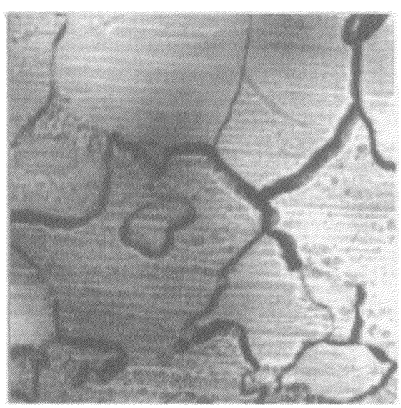

$\varphi=80 \%$

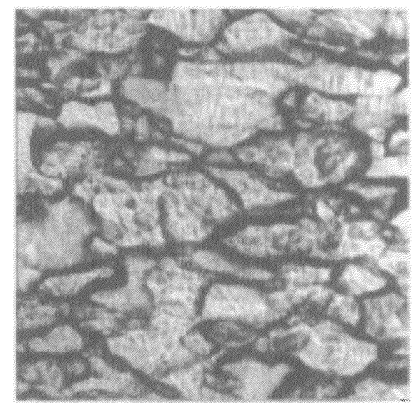

$\varphi=40 \%$

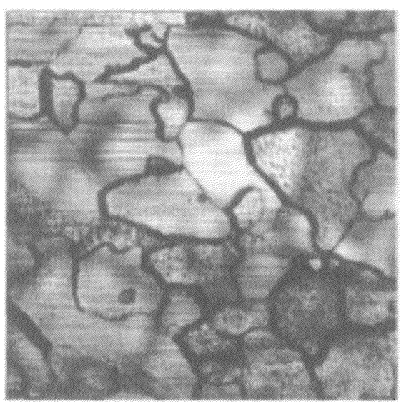

$\varphi=95 \%$

Figure 5 Grain sizes at increasing degrees of rolling, $\mathrm{T}=-35^{\circ} \mathrm{C}$, magn. $80 \mathrm{x}$. 


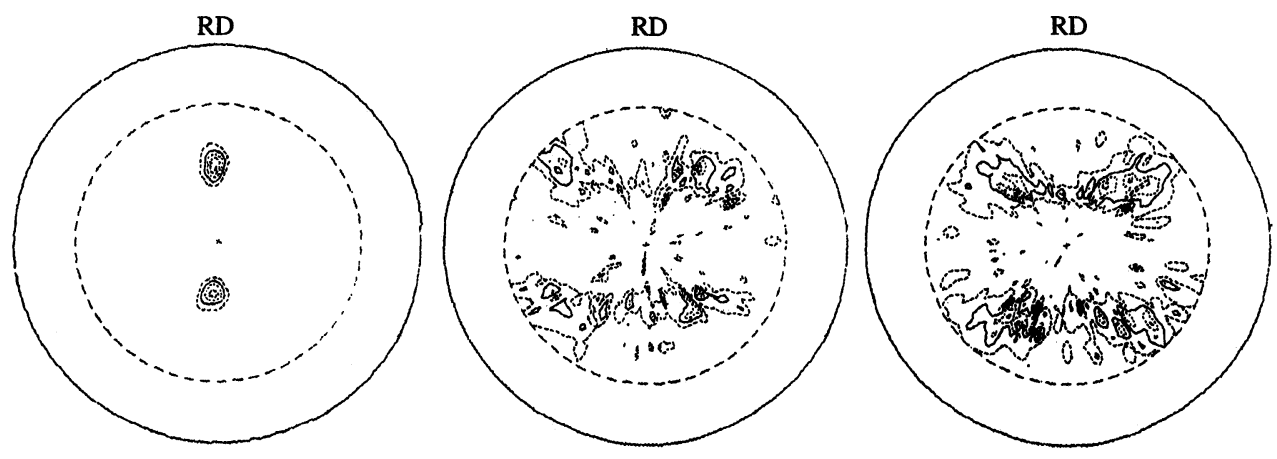

Figure 6 (111)-Pole figures of rolled silver chloride, $\mathrm{T}=25^{\circ} \mathrm{C}$.

was the reason to carry out experiments at the lower temperatures. Cooling down the specimen before each rolling step shifted the beginning of grain enlargement to higher degrees of rolling but it could not prevent it completely.

At a degree of rolling of $20 \%$ the initial texture was still fully present (Figure 6). The ODF in Figure 7 shows that this is a very sharp Goss texture produced by the extrusion process. At $60 \%$ there is a beginning cube texture with the Goss texture still being present. A tilted cube texture like in recrystallized aluminium can also be seen. At $80 \%$ the Goss texture has nearly disappeared.

The cube texture being a typical recrystallisation texture of fcc metals is another indication of dynamic recystallisation. There is good agreement between grain growth, occurrence of the cube texture and vanishing grain elongation all taking place at the same degree of rolling. The pole figures are not very clear due to the large grain sizes at $60 \%$ and $80 \%$. Typical rolling textures could not be obtained due to dynamic recrystallization.

Literature data about recrystallization of silver chloride differ a lot (Weber, 1986; Gmelin, 1971) when starting temperatures and recystallization times are concerned. Specimens were chosen that were reduced by $30 \%$ at room temperature to exclude effects of dynamic recrystallization induced by rolling. The initial grains showed distinct elongation with the length being approximately twice the width. Grain sizes varied from $50 \mu \mathrm{m}$ to $100 \mu \mathrm{m}$. At a recrystallisation temperature of $100^{\circ} \mathrm{C}$ after six minutes the grain structure started to become globular and the size of the grains was reduced by $20 \%$. Globular shape of the grains was achieved after twenty minutes whereas the grain size remained constant until $t=30 \mathrm{~min}$. After that, secondary recrystallization took place which produced very large grains (Figure 8 ) that could be well observed without a microscope after etching.

At a temperature of $120^{\circ} \mathrm{C}$ secondary recrystallization started already after six minutes but the grain size did not exceed $200 \mu \mathrm{m}$ even after exposing the specimens to this temperature for 30 minutes. This size was obtained already after 10 minutes and at the same time the structure became globular. Secondary recrystallization started already after four minutes when a temperature of $150^{\circ} \mathrm{C}$ was applied. The final size was reached after about six minutes but the reduction of the elongation of the grains did not start until $t=15 \mathrm{~min}$. It could of course be expected that secondary recrystallization starts at earlier times when the temperature is raised. Contrary to this is the beginning of the diminishing of the elongation which starts at later times when the temperature 

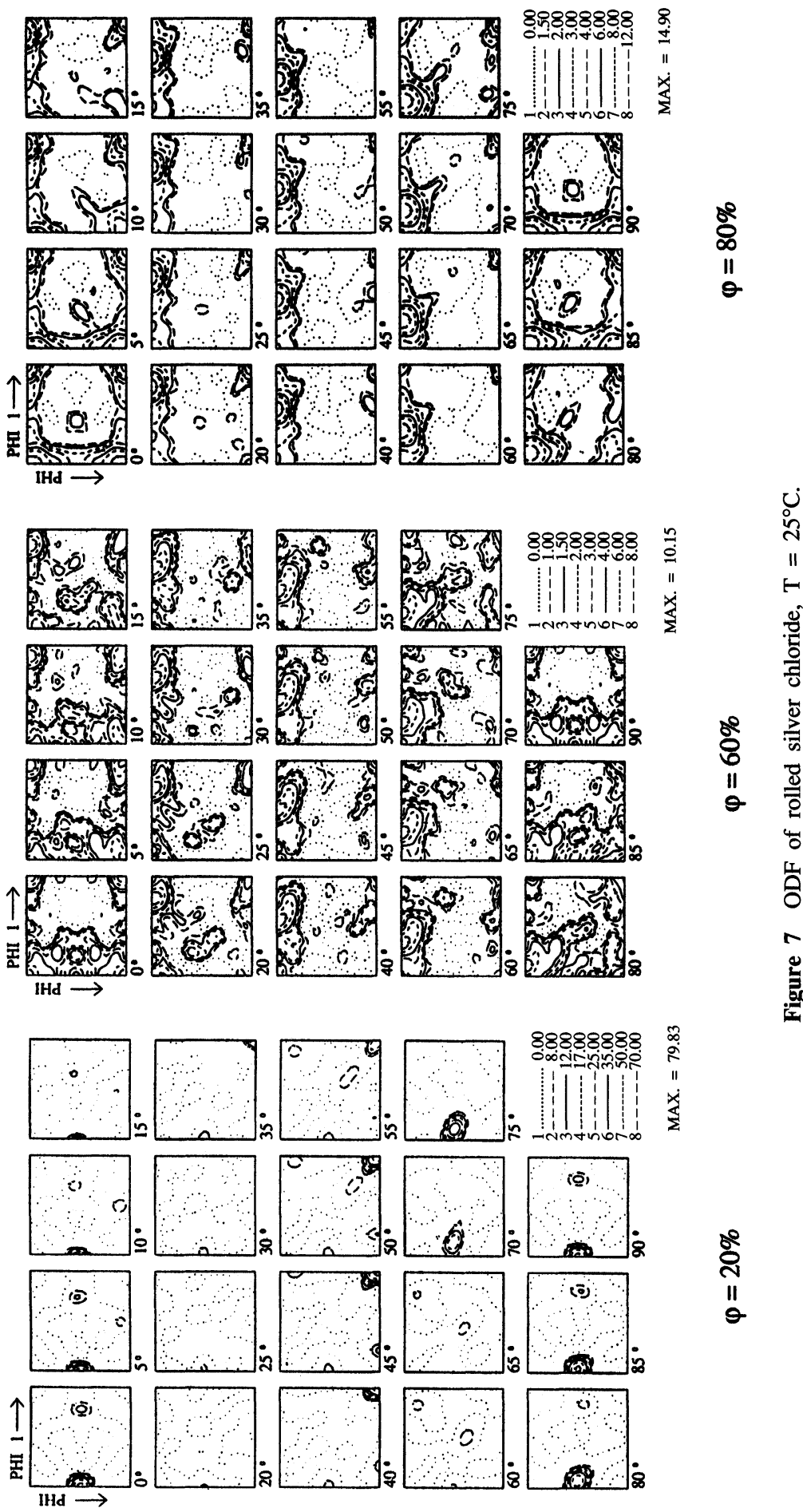

8
0
0
0

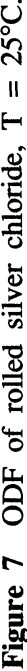

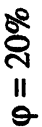

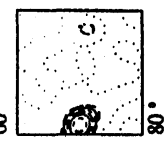




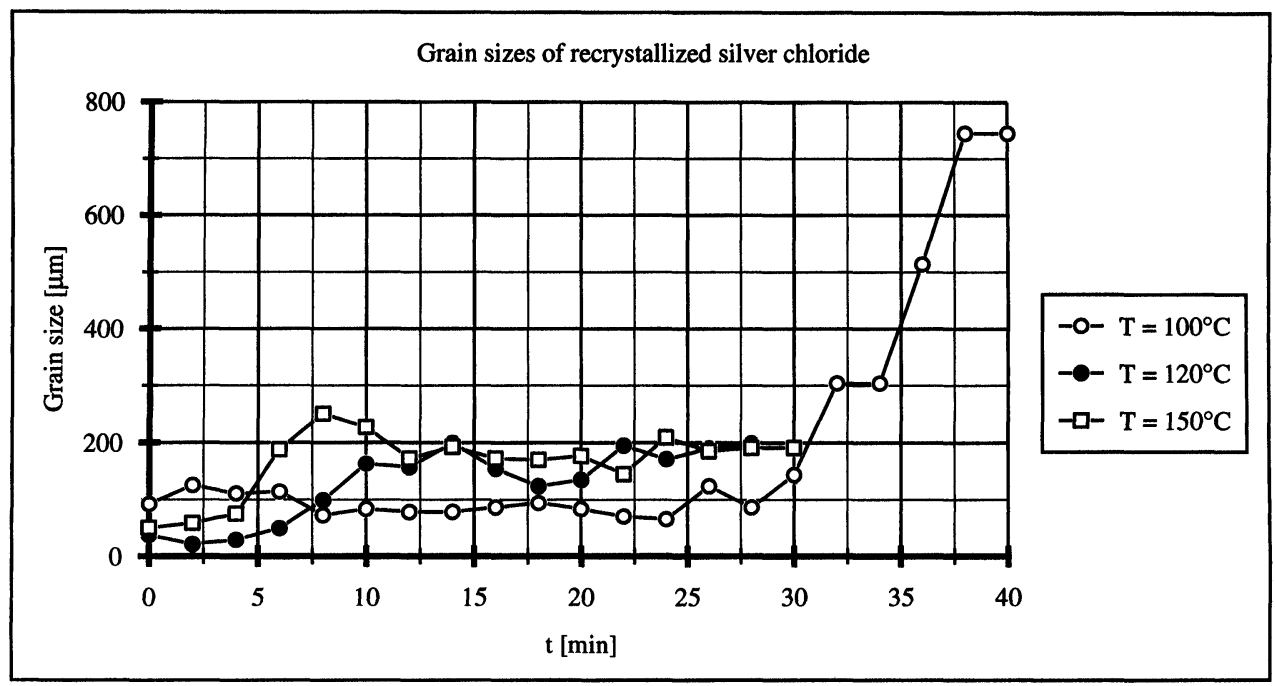

Figure 8 Grain growth of silver chloride at different recrystallization temperatures and $30 \%$ degree of rolling.

is raised. At high annealing temperatures small final grain sizes were obtained. A higher tempe-rature produces coagulation of precipitations (Brickenkamp, 1984). This results in a greater number of grains being able to grow simultaneously and thus producing a smaller final grain size. In a single-phase material this effect can be due to impurities. In the case of silver chloride which has been submitted to visible light there is always coagulated silver present which is probably responsible for this effect.

\section{PHOTOELASTIC EFFECT IN MONOCRYSTALLINE SILVER CHLORIDE}

To be able to relate photoelastic effects and texture it is necessary to quantify the relationship of the stress state and the photoelastic effects, i.e. relative retardation and extinction angle. For this reason single-crystal studies have been carried out. In general, the directions of the principal stress, the principal axes of the indicatrix or of the Fresnelellipsoid, the crystal axes, the directions of the incident wave normal and the orientation of the sample do not coincide. In the present investigations the sample direction, stress direction and the wave normal direction were chosen such that they were the same. In this case one only has to know the orientation of the index-ellipsoid and the crystal orientation in relation to the sample coordinate system. The extinction angle was measured by an ordinary polariscope and the crystal orientation by an x-ray goniometer. The angle between the Fresnel-ellipsoid and the sample depends on the stress tensor, the stress level and the orientation of the specimen. Therefore it is necessary to determine the relative phase retardation. This is achieved by the compensation method of Sénarmont which is a built-in feature of most polariscopes.

A plane-parallel plate of silver chloride in a plane state of stress is to be considered. The plate is cut in an arbitrary way, so there are three coordinate systems to be taken into account. These are denoted as XYZ-crystal coordinates, $X^{\prime} Y^{\prime} Z^{\prime}$-system of principal stresses with $Z^{\text {' }}$ perpendicular to the plate surface and $X^{\prime \prime} Y^{\prime \prime} Z$ " 
system of the optical ellipsoid. The index ellipsoid expressed in the $X^{\prime} Y^{\prime} Z^{\prime}-$ system has the general form

$$
\mathrm{B}_{11}^{\prime} \mathrm{x}^{\prime 2}+\mathrm{B}_{22}^{\prime} \mathrm{y}^{\prime 2}+\mathrm{B}_{33}^{\prime} \mathrm{z}^{\prime 2}+2 \mathrm{~B}_{23}^{\prime} \mathrm{y}^{\prime} \mathrm{z}^{\prime}+2 \mathrm{~B}_{13}^{\prime} \mathrm{x}^{\prime} \mathrm{z}^{\prime}+2 \mathrm{~B}_{12}^{\prime} \mathrm{x}^{\prime} \mathrm{y}^{\prime}=1
$$

For the plane case with $\mathrm{Z}^{\star}=0$ this simplifies to an ellipse of the form

$$
\mathrm{B}_{11}^{\prime} \mathrm{x}^{\prime 2}+\mathrm{B}_{22}^{\prime} \mathrm{y}^{\prime 2}+2 \mathrm{~B}_{12}^{\prime} \mathrm{x}^{\prime} \mathrm{y}^{\prime}=1
$$

The $\mathrm{B}^{\mathrm{i}}{ }_{\mathrm{ii}}$ describe the intersection of the ellipse and the coordinate system whereas $\mathrm{B}_{12}$ is a measure for the angle $\alpha$ which is the optical isocline or the extinction angle.

$$
\tan 2 \alpha=\frac{2 B_{12}^{\prime}}{B_{11}^{\prime}-B_{22}^{\prime}}
$$

The Fresnel-ellipsoid and the stress state, which is an ellipsoid as well, are to be related to each other. This requires a system of 36 stress-optical coefficients $\pi_{\mathrm{ij}}$ in the general case. Because of cubic symmetry only three coefficients remain. With the assumption that the changes of the coefficients $\mathrm{B}_{\mathrm{ij}}^{\mathrm{i}}$ of the ellipsoid are linear functions of the stresses (Krasnov, 1947) these coefficients can be expressed as

$$
\begin{aligned}
& \mathrm{B}_{11}^{\prime}-\mathrm{v}_{0}^{2}=\pi_{11}^{\prime} \sigma_{1}+\pi_{12}^{\prime} \sigma_{2} \\
& \mathrm{~B}_{22}^{\prime}-\mathrm{v}_{0}^{2}=\pi_{12}^{\prime} \sigma_{1}+\pi_{22}^{\prime} \sigma_{2} \\
& 2 \mathrm{~B}_{12}^{\prime}=\pi_{16}^{\prime} \sigma_{1}+\pi_{26}^{\prime} \sigma_{2}
\end{aligned}
$$

Herein the $\sigma_{\mathrm{i}}$ are the principal stresses, $\mathrm{v}_{0}$ is the speed of light in unstressed AgC1 and the $\pi_{\mathrm{ij}}{ }^{6}$ are functions of the three piezooptic coefficients $\pi_{11}, \pi_{12}$ and $\pi_{44}$ in the crystal coordinate system. If these are substituted into eq. (4) and then into eq. (3) $\alpha$ is expressed as a function of the piezooptic coefficients, the principal stresses and the direction cosines of the angles between the crystal and the sample coordinate system all of which are known.

The angle $\alpha$ of the optical isocline gives the planes of polarization of the two waves emerging from the crystal. The relative path difference of these two waves can be measured by a compensation method by adding or subtracting an artificial path difference until extinction occurs for a particular wavelength. When this is done in monochromatic light an accuracy of better than 0.02 fringe orders can be achieved. The path differences as well as the optical isocline depend on the state of stress as was stated earlier. Therefore the relation between the path differences and the state of stress has to be formulated. If we write eq. (4) in the optical coordinate system $X^{\prime \prime} Y$ " $Z$ " we obtain

$$
\begin{aligned}
& \mathrm{B}_{11}^{\prime \prime}-\mathrm{v}_{0}^{2}=\pi_{11}^{\prime \prime} \sigma_{\mathrm{x}^{\prime \prime}}+\pi_{12}^{\prime \prime} \sigma_{\mathrm{y}^{\prime \prime}}+\pi_{16}^{\prime \prime} \tau_{\mathrm{x}^{\prime \prime} \mathrm{y}^{\prime \prime}} \\
& \mathrm{B}_{22}^{\prime \prime}-\mathrm{v}_{0}^{2}=\pi_{12}^{\prime \prime} \sigma_{\mathrm{x}^{\prime \prime}}+\pi_{22}^{\prime \prime} \sigma_{\mathrm{y}^{\prime \prime}}+\pi_{26}^{\prime \prime} \tau_{\mathrm{x}^{\prime \prime} \mathrm{y}^{\prime \prime}} \\
& 2 \mathrm{~B}_{12}^{\prime \prime}=0=\pi_{16}^{\prime \prime} \sigma_{\mathrm{x}^{\prime \prime}}+\pi_{26}^{\prime \prime} \sigma_{\mathrm{y}^{\prime \prime}}+2 \pi_{26}^{\prime \prime} \tau_{\mathrm{x}^{\prime \prime} \mathrm{y}^{\prime \prime}}
\end{aligned}
$$

The $\mathrm{B}_{\mathrm{ii}}$ represent the speeds of the polarized light waves emerging from the crystal. According to Krasnov (1947) they can be expressed as 


$$
\begin{aligned}
& \mathrm{B}_{11}^{\prime \prime}=\mathrm{v}_{0}^{2}\left(1+\frac{\mathrm{p}}{\mathrm{v}_{0}^{2}} \sigma_{\mathrm{x}^{\prime \prime}}+\frac{\mathrm{q}}{\mathrm{v}_{0}^{2}} \sigma_{\mathrm{x}^{\prime \prime}}\right) \\
& \mathrm{B}_{22}^{\prime \prime}=\mathrm{v}_{0}^{2}\left(1+\frac{\mathrm{q}}{\mathrm{v}_{0}^{2}} \sigma_{\mathrm{x}^{\prime \prime}}+\frac{\mathrm{r}}{\mathrm{v}_{0}^{2}} \sigma_{\mathrm{x}^{\prime \prime}}\right)
\end{aligned}
$$

where $\mathrm{p}, \mathrm{q}$ and $\mathrm{r}$ are functions of the piezooptic coefficients and the orientation of the optical coordinate system in relation to the crystallographic system. The speeds of the light waves then have to be expressed in terms of refraction indices. Transforming stresses back into principal stresses leads to the conditional equation (7) for the relative optical path difference $\delta$.

$\delta=\mathrm{d} \frac{\mathrm{V}}{2 \mathrm{v}_{0}^{3}}\left\{\left[(\mathrm{p}-\mathrm{q}) \cos ^{2} \alpha-(\mathrm{r}-\mathrm{q}) \sin ^{2} \alpha\right] \sigma_{1}+\left[(\mathrm{p}-\mathrm{q}) \sin ^{2} \alpha-(\mathrm{r}-\mathrm{q}) \cos ^{2} \alpha\right] \sigma_{2}\right\}$

$\mathrm{V}$ is the speed of light in vacuum and $\mathrm{d}$ is the thickness of the sample. With these relations the plane state of stress can be calculated from photoelastic measurements or on the other hand photoelastic constants of the material can be calibrated.

\section{EXPERIMENTAL STUDY OF SINGLE CRYSTALS UNDER TENSILE STRESS}

The silver chloride single crystals that were used were $30 \mathrm{~mm}$ long, $10 \mathrm{~mm}$ wide and $3 \mathrm{~mm}$ thick. Small clamps to hold the specimens were constructed. They had the special feature that contact pressure was proportional to the tensile load in order not to deform the fixing points more than was necessary. These were mounted into a loading frame with a mechanical load-sensing device. The load was increased in steps of $50 \mathrm{~N}$ to a limit of $15 \mathrm{~N} / \mathrm{mm}^{2}$. One of the samples cracked when reaching this limit. Plastic flow started at $3-5 \mathrm{~N} / \mathrm{mm}^{2}$.

The loading frame with the specimens was placed in a magnifying projection polariscope and extinction angles and relative retardation measured. While the load was increased shearing of the cross section of the sample occurred. Figure 9 shows the curves of true stress versus relative retardation for three samples. They show good linearity even in the plastic range which begins at a stress of less than $5 \mathrm{~N} / \mathrm{mm}^{2}$.

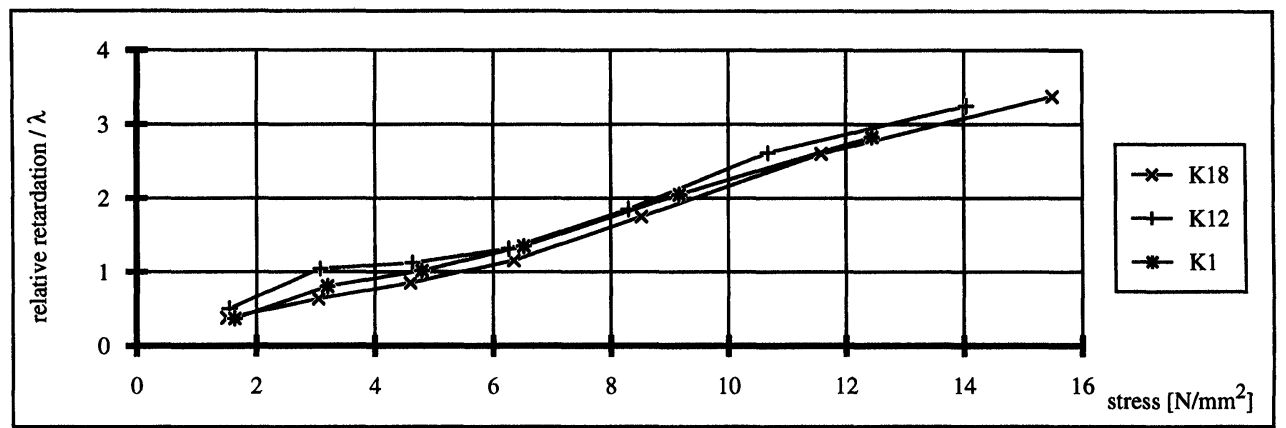

Figure 9 Relative retardation at $\lambda=546 \mathrm{~nm}$ versus stress of specimens K1, K12 and K18. 
Table 1 Values of the piezooptical constants. (Units $=\mathrm{mm}^{2} / \mathrm{N}$ )

\begin{tabular}{lccr}
\hline & West/Makas & Goodman/Sutherland & \multicolumn{1}{c}{ Gieleßen } \\
\hline$\pi_{11}-\pi_{12}$ & $-5,62 \cdot 10^{-6}$ & $-9,4 \cdot 10^{-6}$ & $-5,72 \cdot 10^{-6}$ \\
$\pi_{44}$ & $8,89 \cdot 10^{-6}$ & $12 \cdot 10^{-6}$ & $7,97 \cdot 10^{-6}$ \\
\hline
\end{tabular}

The question now is whether these measurements are in agreement with the theory given in chapter 5 . From the above equations it can be seen that the three piezooptical or the three elastooptical constants and certain transformation matrices are needed. The orientation of Specimen K12 was measured by an X-ray goniometer to be $\overline{2}, 1,8)$ $[\overline{3}, 1, \overline{1}]$. The required optical constants have only been determined twice before by photoelastic measurements. The values are given in Table 1.

It is not possible by photoelastic means to separate $\pi_{11}$ and $\pi_{12}$. To obtain the single constants measurements of the absolute retardation have to be carried out with an interferometer. From these constants, the crystal orientation and the applied stress a theoretical isochromatic fringe order can be calculated and compared with the measured fringe order obtained with a polariscope and linear polarized light. The light source was a white light lamp with a metal interference filter of a wave-length of $589 \mathrm{~nm}$.

Until a stress level of about $3 \mathrm{~N} / \mathrm{mm}^{2}$ the measurements were in good agreement with the theoretical values calculated from our constants and those of West / Makas (1948). Above this level plastic flow began and the fringe order increased only a little until $6 \mathrm{~N} / \mathrm{mm}^{2}$ were reached. At even higher stress levels the effect became linear again. This was due to a change of orientation of the crystal starting at the beginning of plastic flow. Birefringent bands as described above could be clearly seen to appear at this stress level and spreading over most of the specimen until $6 \mathrm{~N} / \mathrm{mm}^{2}$ were reached. Above this level the change of orientation was complete and the photoelastic effect became linear again.

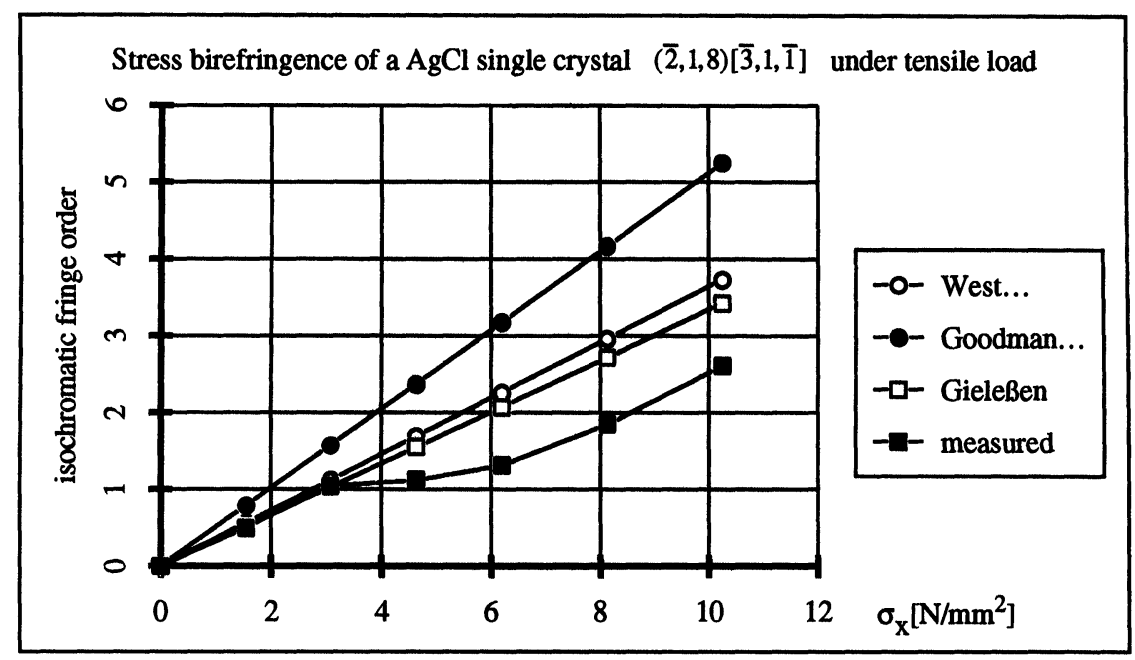

Figure 10 Photoelastic measurement vs. theory. 


\section{OPTICAL ANISOTROPY OF SILVER CHLORIDE}

The orientation of the grains is reflected by the state of refraction. The relation between the indicatrix or the Fresnel-ellipsoid and the state of stress and strain is characterized by the elastooptical or piezooptical constants respectively. In general this can be written as

$$
\begin{array}{ll}
\Delta \mathrm{B}_{\mathrm{ij}}=\pi_{\mathrm{ijk} 1} \sigma_{\mathrm{k} 1} & \pi_{\mathrm{ijkl}}=\text { piezooptical constants } \\
\Delta \mathrm{B}_{\mathrm{ij}}=\mathrm{p}_{\mathrm{ijrs}} \varepsilon_{\mathrm{rs}} & \mathrm{p}_{\mathrm{ijrs}}=\text { elastooptical constants } \\
\mathrm{p}_{\mathrm{ijrs}}=\pi_{\mathrm{ijk} 1} \mathrm{c}_{\mathrm{klrs}} & \mathrm{c}_{\mathrm{klrs}}=\text { elastic constants }
\end{array}
$$

where $\pi_{\mathrm{ijk} 1}$ and $\mathrm{p}_{\mathrm{ijk} \mathbf{1}}$ denote fourth rank tensors describing the material property with 81 components in the general case. In order to take the cubic crystal symmetry into account, it is more convenient to use the matrix representation $\pi_{\mathrm{mn}}, \mathrm{p}_{\mathrm{mn}}$ with $\mathrm{m}, \mathrm{n}$ running from 1 to 6 (Nye, 1957). The remaining constants are $\pi_{11}, \pi_{12}$ and $\pi_{44}$ for cubic $\mathrm{m} 3 \mathrm{~m}$ material.

These three remaining piezooptical constants for cubic material have been determined using a simple interferometric setup. Only few measurements have been made until now and as the interferometric fringe orders were not very distinct further investigations must be carried out. From the preliminary values of the constants a spatial representation surface (Figure 11) can be calculated (Nye, 1957) using equation 9:

$$
\pi_{\overrightarrow{\mathrm{r}}}=\pi_{11}-2\left(\pi_{11}-\pi_{12}-\frac{1}{2} \pi_{44}\right)\left(1_{1}^{2} 1_{2}^{2}+1_{2}^{2} 1_{3}^{2}+1_{3}^{2} 1_{1}^{2}\right) ; \overrightarrow{\mathrm{r}}=\left\{1_{1}, 1_{2}, 1_{3}\right\}
$$

The directions of the maximum values are the cube axes. As this diagram shows the piezooptic constant the photoelastic effect (the isochromatic fringe order) which is always a differential effect is not represented herein.

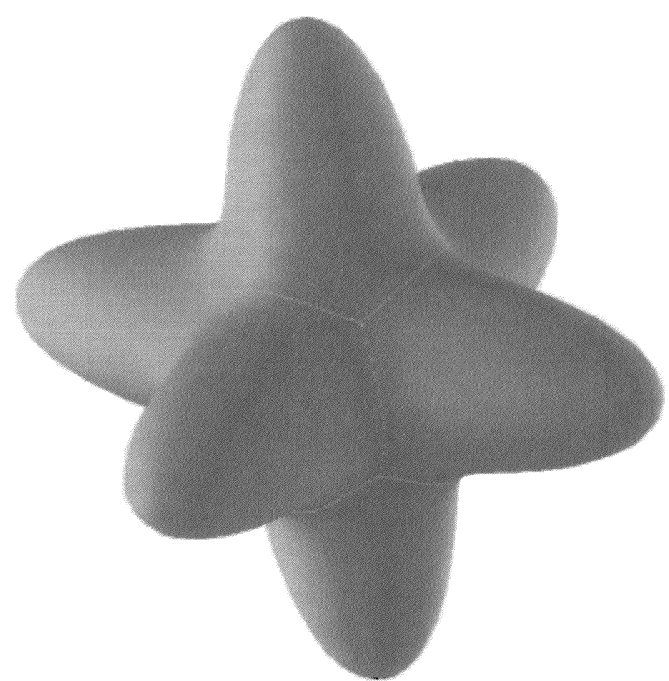

Figure 11 Orientation dependence of the piezooptical constant of silver chloride. 
Table 2 Values of the piezooptical constants of $\mathrm{AgCl}$.

\begin{tabular}{l} 
Piezooptical constants of silver chloride \\
$\pi_{11}=4.38 \times 10^{-6} \mathrm{~mm}^{2} / \mathrm{N}$ \\
$\pi_{12}=1.01 \times 10^{-5} \mathrm{~mm}^{2} / \mathrm{N}$ \\
$\pi_{44}=7.97 \times 10^{-6} \mathrm{~mm}^{2} / \mathrm{N}$ \\
\hline
\end{tabular}

In the simple case of uniaxial load in [001]-direction the relative retardation measured normal to the sample surface and the stress direction is calculated from the piezooptical constants as

$$
\mathrm{s}=-\frac{1}{2} \mathrm{dn}_{0}^{3}\left(\pi_{11}-\pi_{12}\right) \sigma
$$

with $\mathrm{n}_{0}$ being the isotropic index of refraction and $\mathrm{d}$ being the thickness of the sample. When related to the wavelength of the light that was used this results in the isochromatic fringe order.

\section{PHOTOELASTIC ANISOTROPY IN POLYCRYSTALLINE MATERIAL}

The next step is to show whether there are visible effects of optical anisotropy in polycrystalline silver chloride at all. A sample was rolled with a reduction of $60 \%$ and then partially recrystallized in a way that coarse grain was produced. The sample was placed in a polariscope and tensile stress applied (Figure 12).

The different grains show up in different colours (= isochromatic fringe orders) depending on the orientation of the grains. Grain boundaries are not very distinct in the photoelastic image due to partial overlapping in the direction of view.

The directional dependancy of the photoelastic effect can be shown with a textured specimen loaded in various directions. Samples were rolled with a reduction of $60 \%$ again and specimens cut out of them at angles of $0^{\circ}, 45^{\circ}$ and $90^{\circ}$ to the rolling direction. These were subjected to tensile stress and relative retardation was measured (Table 3 ).

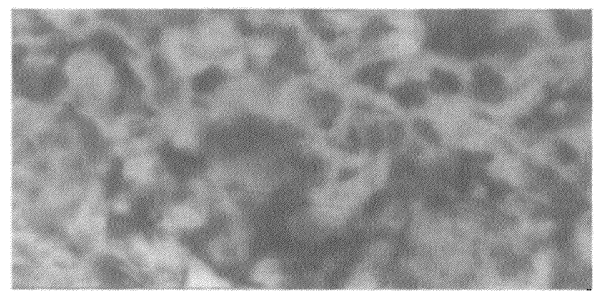

(a)

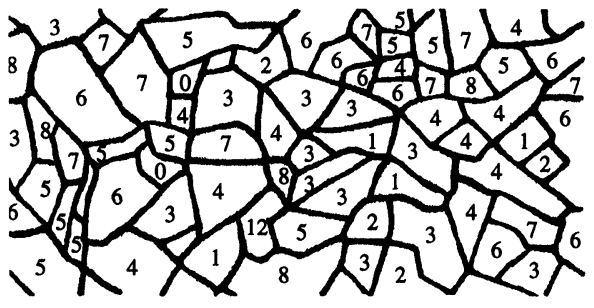

(b)

Figure 12 Isochromatic fringe order in polycrystalline specimen under tensile load (unit $=1 / 10$ ).

Table 3 Relative retardation, measured in different directions.

$$
\begin{aligned}
& \mathrm{s}_{0^{\circ}}=2,78 \mathrm{~nm} / \mathrm{N} \\
& \mathrm{s}_{45^{\circ}}=3,67 \mathrm{~nm} / \mathrm{N} \\
& \mathrm{s}_{90^{\circ}}=2,27 \mathrm{~nm} / \mathrm{N}
\end{aligned}
$$




$$
\begin{aligned}
& \mathrm{s}=\mathrm{s}_{0}+\mathrm{s}_{2} \cos 2 \alpha+\mathrm{s}_{4} \cos 4 \alpha \\
& \mathrm{s}_{0}=\frac{1}{4}\left[\frac{1}{\mathrm{~s}_{90^{\circ}}}+\frac{1}{\mathrm{~s}_{0^{\circ}}}+\frac{2}{\mathrm{~s}_{45^{\circ}}}\right]=0,335 \\
& \mathrm{~s}_{2}=\frac{1}{2}\left[\frac{1}{\mathrm{~s}_{90^{\circ}}}-\frac{1}{\mathrm{~s}_{0^{\circ}}}\right]=0,040 \\
& \mathrm{~s}_{4}=\frac{1}{4}\left[\frac{1}{\mathrm{~s}_{90^{\circ}}}+\frac{1}{\mathrm{~s}_{0^{\circ}}}-\frac{2}{\mathrm{~s}_{45^{\circ}}}\right]=0.063
\end{aligned}
$$

The angular dependence of the retardation can be expressed by eq. (11). From the measurements the isotropic part and the parts of two-fold and four-fold symmetries (eq. 12) were calculated (Bunge, 1982) in the plane of rolling. In the polar diagram (Figure 13) the anisotropy of the photoelastic effect in the sample plane can be clearly seen. The coefficients and the diagram in Figure 13 show that the isotropic part is dominant and that the direction of the largest effect coincides with the rolling direction.

\section{CONCLUSIONS}

Progresses in the application of the technique of photoelasticity have been presented in this paper. For the first time metal working processes have been used to create mechanical and optical anisotropy in a doubly refracting material in order to simulate mechanical anisotropy in metal parts. When the photoelastic constants are measured with better accuracy it will be possible to average them with the coefficients of the orientation distribution function and to obtain the macroscopic photoelastic effect of textured specimens. With known relations between texture and photoelastic effect representation laws can be set up which then permit to relate the photoelastic effect with the state of stress in the real metal part with known texture.

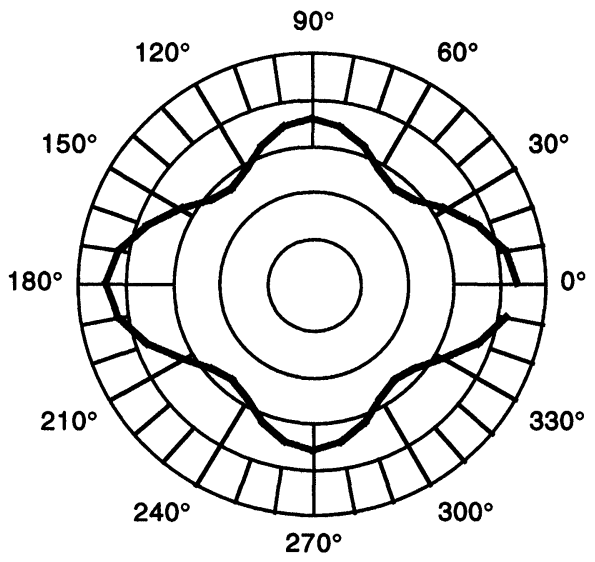

Figure 13 Optical anisotropy of rolled specimens. 


\section{References}

Brickenkamp, W. (1984). Kornvergrößerung, sekundäre und tertiäre Rekristallisation. In Rekristallisation metallischer Werkstoffe, edited by G. Gottstein, pp. 83-98, Oberursel: Deutsche Gesellschaft für Metallkunde.

Bunge, H. J. and Esling, C. (1982) Mean values of physical properties. In Quantitative Texture Analysis, edited by H. J. Bunge, C. Esling, pp. 383-406, Oberursel: Deutsche Gesellschaft für Metallkunde.

Gmelin (1971). Handbuch der anorganischen Chemie, Ag[B1], 6.2.3.7.3., pp. 378-380, Weinheim: Verlag Chemie $\mathrm{GmbH}$.

Goodman, E. and Sutherland, J. G.(1953). Elasto-plastical stress-optical effect in $\mathrm{AgCl}$ single crystals. J. Appl. Phys., 24, 5, 557-584.

Krasnov, V. M. (1947) On determination of stress in cubic crystals by means of the optical method. Ucenye Zapiski LGU, ser. mat.-mech., 13, no. 87, 97-114 (in russian).

Nye, J. F. (1949). Plastic deformation of silver chloride (Part I). Proc. Royal. Soc. London, A198, $190-204$.

Nye, J. F. (1957). Physical properties of crystals, reprinted 1967, London: Clarendon Press.

Stepanov, A. V. and Zhitnikov, R. A. (1956a). An optical method of investigation of averaged stressed states in small-grained polycrystals, part I: Preparation of small-grain polycrystal specimens of silver chloride. Soviet Physics - Technical Physics, 4, 754-761.

Stepanov, A. V. and Zhitnikov, R. A.(1956b). An optical method of investigation of averaged stressed states in small-grained polycrystals, part III: Photoelastical(piezooptical) properties of polycrystalline silver chloride. Soviet Physics - Technical Physics, 4, 769-776.

Tamman, G. (1932). Naturwissenschaften, 20, 958.

Weber, H. J. (1986). Eigenschaften des spannungsoptisch aktiven Modellwerkstoffes Silberchlorid und seine Anwendung auf die Untersuchung von Spannungszuständen mit teilweise plastischer Verformung. Dissertation, University of Clausthal.

West, C. D. and Makas, A. S. (1948). Technical crystals with abnormally large stress birefringence. Polaroid Corporation, Cambridge 39, Massachusetts. 